\title{
Seeing through the mist: an evaluation of an iteratively designed head-up display, using a simulated degraded visual environment, to facilitate rotary-wing pilot situation awareness and workload
}

\author{
Neville A. Stanton ${ }^{1} \cdot$ Katherine L. Plant $^{1} \cdot$ Aaron P. Roberts $^{1} \cdot$ Craig K. Allison $^{1}$ (D) $\cdot$ Mike Howell $^{1}$
}

Received: 22 May 2019 / Accepted: 19 August 2019 / Published online: 24 August 2019

(c) The Author(s) 2019

\begin{abstract}
Degraded visual conditions present a great challenge to rotary-wing aircraft. These conditions can obscure cues used to interpret speed, location and approach. With such cues obscured, pilots must rely on in-cockpit instrumentation, increasing workload, whilst reducing situation awareness. When operating within degraded visual conditions, pilots require easy access to flight critical information, presented in a way that minimises additional workload and maximises situation awareness. One technology that can be beneficial within such conditions is a head-up display (HUD). This study explores the impact of an iteratively designed HUD on pilots' workload and situation awareness during the safety-critical descent and landing flight phases, during both clear and degraded visual conditions across a series of simulated trials. Results suggest that access to the HUD facilitated pilot awareness, whilst maintaining workload in all conditions. Results support the view that HUDS are beneficial to rotary-wing pilots, particularly in degraded visual environments.
\end{abstract}

Keywords Rotary-wing $\cdot$ Helicopters $\cdot$ Workload $\cdot$ Situation awareness $\cdot$ Head-up displays

\section{Practitioners summary}

Rotary-wing operations in degraded visual environments are a great challenge. This paper presents an experimental simulation study with rotary-wing pilots to assess how a novel future HUD design can support operations in such conditions. Results demonstrate the potential of such in-cockpit technologies.

Electronic supplementary material The online version of this article (https://doi.org/10.1007/s10111-019-00591-2) contains supplementary material, which is available to authorized users.

Craig K. Allison

Craig.Allison@soton.ac.uk

Transportation Research Group, Faculty of Engineering and Environment, University of Southampton, Boldrewood Innovation Campus, Southampton SO16 7QF, UK

\section{Introduction}

Despite modern aviation technology, weather, specifically low visibility, remains a leading contributor to both flight delays and, more critically, airline accidents worldwide (Allan et al. 2001; Sridhar and Swei 2006; Federal Aviation Administration 2001). Degraded visual conditions are of significant concern to rotary-wing operations, wherein it is a key contributor to fatalities (De Voogt and Van Doorn 2007). The operational benefits of rotary-wing aircraft, including low-altitude flight, vertical take-off/landing and the capacity to hover with zero ground speed mean they are often used for flights to remote, unscheduled locations including flight to unimproved landing sites without aviation-related ground supportive infrastructure (Baker et al. 2011; Swail and Jennings 1999). Of central concern with the use of rotary-wing aircraft is that recorded accident rates are significantly greater than fixed-wing aircraft counterparts (Doehler et al. 2009).

Flight within degraded visual conditions is associated with a dramatic increase in pilots' workload, alongside a noticeable increase in the complexity of the overall flight task. Combined, these factors result in a potential deterioration of the cognitive capacity of the pilot (Shappell and 
Wiegmann 2004). This deterioration increases the likelihood that errors can occur (Sneddon et al. 2013), negatively impacting safety. The aim of the current work is to develop and test novel head-up display (HUD) technologies that can be used to facilitate rotary-wing flight in degraded visual conditions in order to increase safe operational capabilities. This work is a continuation of previous HUD development work seeking to improve rotary-wing pilots' situation awareness and optimise pilot workload, especially within degraded visual conditions (Stanton et al. 2016, 2017, 2019). Although HUDs have been well researched and documented within fixed-wing aircraft, their use in rotary-wing aircraft is limited and specific research is required to understand whether the insights gained from work within fixed-wing is applicable to this domain. Although industrial research into HUD use within rotary-wing aircraft has been conducted, this research has largely been restricted to industry-led research and this topic remains under-represented within publically accessible, peer-reviewed, academic, literature.

During flight in clear visibility the majority of the information rotary-wing pilots use to fly is visual, with even the most basic manoeuvres requiring maintenance of visual awareness to at least three external reference points to monitor altitude and position. In clear visual conditions pilots anticipate the dynamics of the aircraft; conducting routine flight parameter sampling, as they fly proactively (Prinzel et al. 2004). The cockpit instrument scanning behaviour of pilots supports the notion of confirmatory information 'check', whereby time is dedicated to the sampling of information only if an unexpected discrepancy is observed (Wickens 2002). Supporting the importance of visual cues, one analysis of helicopter accidents near offshore drilling platforms identified awareness of obstacles at the destination; sufficiency of visual cues for approach; stability of visual cues and sufficiency of visual aids for landing as the primary performance factors (Nascimento et al. 2013).

Situation awareness can be defined as the perception of elements in the environment at a specific time and space, comprehension of their meaning and projection of their future status (Endsley and Jones 2012). This knowledge facilitates understanding of the current situation (Bell and Lyon 2000). Considered within the context of rotary-wing operations, pilot situation awareness is the construction of an understanding of the current situation, based on the extraction of relevant information from the environment and prior experience (Plant and Stanton 2012). When flying at low altitude, such as during descent and landing, visual information from the outside world greatly informs pilot situation awareness (Doehler et al. 2009), providing cues such as the physical geometry of terrain and objects, texture density and the rate of visual flow (Foyle et al. 1992). In degraded visual conditions pilots can no longer rely on visual information from the external environment.
As such, the ability to fly in an anticipatory fashion is reduced (Doehler et al. 2009) and overall situation awareness constrained. Pilots subsequently rely on reactionary pursuit control where cockpit instrumentation is used to generate understanding of the external environment (Snow and French 2002; Harris 2011; Wickens 2002). As a consequence of this shift, and pilots' greater reliance on cockpit instrumentation, cognitive demand and therefore their subsequent workload is increased (Prinzel et al. 2004).

The aim of future cockpit technologies is to optimise pilot workload and improve situation awareness, particularly in degraded visual conditions (Harris 2011; Melzer 2012). Despite technological development and the improvements compared to older "one indicator/one instrument" cockpits, the use of rotary-wing cockpit technologies remains a challenge (Harris 2011). The landing task can be actively supported by the use of an instrument landing system (ILS), providing accurate lateral guidance and glide slope allowing an aircraft to reach the runway at a predefined touchdown point (Geise et al. 2008). In many cases, ILS systems can be used to direct autopilot systems so that approach and landings are automatic and do not require manual input. In addition, fixed-wing aircraft can be equipped with enhanced flight vision systems to allow greater visibility to crew during periods of high workload such as take-off and landing. These technologies are, however, not always available to rotary-wing pilots as these systems are not available at the unimproved landing sites typically serviced by such aircraft. Alternative systems are therefore needed to support rotary-wing pilots during descent and landing phases of flight.

One piece of technology that can be of significant benefit in this endeavour is a head-up display (HUD, Stanton et al. 2019). A HUD is a glass-mounted panel in the pilots' near visual field that displays flight information, typically 2D traditional flight references (e.g. airspeed, altitude and power) and a representation of the outside environment (Swail and Jennings 1999; Thomas and Wickens 2004; Prinzel et al. 2004). When presenting a display that aligns with the external environment, this is often referred to as conformal symbology (Ververs and Wickens 2000). Due to this enhanced vision system, a HUD allows the pilot to fly 'eyes out' rather than switching attention to head-down displays (HDDs) inside the cockpit. Conformal symbology provides a detailed, realistic representation of the terrain in front of the aircraft and potential obstacles present at low altitude (Thomas and Wickens 2004). A third application of the HUD is the presentation of an integrated graphic flight path representation, often termed "Highway in the Sky' (HITS, Thomas and Wickens 2004). The primary objective of the HITS is to facilitate the pilot's ability to successfully aviate and navigate (Alexander et al. 2003), with previous research demonstrating that the use of HITS can facilitate 
the descent and landing phases of rotary-wing operations (Stanton et al. 2019).

The presentation of information in a way that does not require the pilot to divert visual attention and cognitive resources into the cockpit away from external events and primary flight references such as possible with a HUD has been documented to reduce workload and increase situation awareness (Snow and Reising 1999; Ververs and Wickens 1998; Snow and French 2002). The presentation of synthetic environmental information in HUDs compared to HDDs has also been shown to improve pilot flight performance (Prinzel et al. 2004). Conformal symbology leads to faster detection response to changes in symbology and improved flight path tracking accuracy (Fadden et al. 1998; Snow and French 2002). HITS lead to increased maintenance of lateral and vertical flight path awareness (Williams et al. 2001) and facilitate pilot performance during descent and landing (Stanton et al. 2019). In addition, the inclusion of synthetic terrain features within a HUD significantly improves situation awareness (Snow and Reising 1999). Real flight studies have found that conformal symbology provides improved path control and situation awareness in terrain-challenged operating environments (Prinzel et al. 2002). Whilst access to a HUD is useful for aiding and facilitating the completion of anticipated events, Fadden et al. (1998) note that the detection of unexpected events may be impeded due to the effect of attentional tunnelling. This occurs when attention is allocated to a particular channel of information for longer than optimal, resulting in other relevant information, for example from the external environment or tasks being neglected (Wickens and Alexander 2009; Snow and French 2002).

It should be noted that HUDs are not the only option for display of novel information within rotary-wing cockpits. Synthetic vision systems can also utilise head-worn devices (HWDs), including goggles and helmets (Bailey et al. 2004). Such devices, whilst typical within military service, indeed HWDs have utilised since the 1980s within the military (Rash 2000), are very rare within the civil domain (Arthur et al. 2017). Although, as Arthur et al. (2017) posit, the increased technological capability of HWDs and the increasing transition away from full helmets to glasses based displays offer tremendous future opportunity, a decision was made within the current research programme to only consider HUD displays, which would be more familiar to civilian rotary-wing pilots.

Developing a HUD, specifically supporting rotary-wing operations, that improves situation awareness, optimises workload and reduces the likelihood of attentional tunnelling, especially for use during degraded visibility is a great challenge. An overly cluttered HUD can be detrimental to pilot situation awareness, particularly when task-irrelevant information is presented in demanding situations (Yeh et al.
2003). The current study explores the extent to which a novel HUD design facilitates rotary-wing pilot situation awareness during both descent and landing phases. Across four simulated trials, participants were required to fly, descend and land at a designated location, in both clear and degraded visual conditions, either with or without access to the HUD.

Based on previous work, the following hypotheses can be generated.

1. Access to the HUD, will increase pilot situation awareness during flight in degraded visual environments (Stanton et al. 2017, 2019).

2. Access to the HUD, will reduce pilot workload during flight in degraded visual environments (Stanton et al. 2017, 2019).

3. Access to the HUD will not have a negative effect on pilots' situation awareness or workload during flight in clear visual conditions (Stanton et al. 2017, 2019).

It should be noted that further hypotheses were initially generated with the intention of directly exploring pilot performance, including extensive analysis of the flight paths flown for the different HUD and weather conditions. Whilst all participants completed the descent and landing tasks explored within the current study, flight path data was not successfully captured and hence could not be analysed. As a consequence, this paper explores participants' self-reported situation awareness and workload data, independent of flight performance.

\section{Method}

\subsection{Design}

The study employed a $2 \times 2 \times 2$ within-subjects design. The independent variables were weather (clear or degraded visual environment), display (with or without HUD) and flight phase (phase of descent or final landing phase).

Dependant variables were pilot's subjective situational awareness, landing confidence and subjective workload.

\subsection{Participants}

Eighteen male participants, aged 34-65 years ( $M$ 44.37, SD 8.78) completed this study. All participants were qualified rotary-wing pilots with varying rotary-wing flight hours experience ( $M$ 3804, SD 3468) who had all flown within a year of the study. Participants were recruited using posters disseminated at local airfields. The Research Ethics Committee at the University of Southampton granted ethical approval for this study. Whilst the lack of female participants may be considered a limitation, only $3 \%$ of UK pilots 
currently employed are female (McCarthy et al. 2015). Due to the overwhelming dominance of currently active male pilots and the relatively small sample of participants, the lack of female participants was not viewed as a significant weakness.

\subsection{Equipment and materials}

\subsubsection{Flight simulator}

The study was conducted using a portable desktop-based flight simulator. The portable desktop flight simulator consisted of a laptop computer that displayed the HDD, and three monitors mounted on a stand to raise the height above the laptop and flight controls. The three monitors displayed the simulated flight environment and the HUD. The simulated environment ran using Lockheed-Martin Prepar3D (previously Microsoft flight simulator software). The flight scenario was located over a helipad at Norfolk naval base, Virginia, USA, using the Eurocopter flight model. Prepar3D software is highly customisable and allowed the required weather conditions to be simulated. In the clear sky conditions, the clear weather setting was selected, allowing pilots a considerable view. In the degraded visibility conditions, application of the fog setting reduced visibility to approximately $0.5 \mathrm{~km}$. The flight controls were the rotary-wing ProFlight Trainer evolution control system, consisting of the anti-torque pedals, collective and cyclic. The flight controls connected to the flight simulator via a USB connection.

\subsubsection{Head-down display}

The HDD was displayed to the pilots via the laptop and was available in all four conditions, aligned with the 2D display on the HUD. The HDD consisted of the standard 2D flight instruments found on the primary flight display, including:

- Conformal compass including a heading read out and way point marker. The way point is set to the landing site and will guide the pilot back to the landing site if visual references are lost.

- Direction to go arrow appears on the compass tape if the pilot is not in sight of the airfield, guiding the pilot back to the flight path via the shortest distance (i.e. left or right turn).

- Torque gauge with colour coded percentage readout (red at $100 \%$, amber at $80-99 \%$, black below $80 \%$, amber below $40 \%$, red below $30 \%$ ).

- Airspeed tape (in knots).

- Attitude indicator including gull wing horizon line and roll indicator.
- Vertical speed tape using an inverse logarithmic scale to give more accuracy at lower speeds to assist with hovering.

- Altitude tape.

- Wind direction (orients around a symbol of the aircraft and is also included on the compass tape) and a wind strength indicator. For all conditions, wind speed was limited to not exceed three knots.

- Ground speed (knots).

\subsubsection{Head-up display}

The HUD was simulated as an overlay on the out of the window view and contained the same 2D flight metrics contained in the HDD. In addition, to assist with the landing task the HUD included:

1. HITS flight path markers, which consisted of blue semicircles and stilts representing the flight path to fly to approach over the helipad (Fig. 1). Above and at each side of the semi-circle, markers were included that represented the edge of the full circle. This provided additional visual cues for the pilot and acted as a guide marker if they drifted to the side or above the main semicircle. Each semi-circle was positioned $0.5 \mathrm{~nm}$ from each other.

2. Drift indicators: one drift indicator was associated with the ground speed indicator (Fig. 2). The drift indicator consisted of a dial indication of the lateral $X Y$ directional vector (with respect to the aircraft's heading). This dial rotated to the vectors angle and changed colour to provide an indication of drift: green represented forward movement $\left(-45^{\circ}\right.$ to $\left.45^{\circ}\right)$, amber represented sideways movement (either $-45^{\circ}$ to $-135^{\circ}$ or $45^{\circ}-135^{\circ}$ ) and red represented backwards movement $\left(-135^{\circ}\right.$ to $\left.135^{\circ}\right)$. The second drift indicator appeared at low speeds (below 30 knots) to assist with drift during the final hover phase. This indicator is a cross hair attached to a vector line. An upward pointing vector line represented forward movement, downward represented backwards movement and out to either side represented left or right drift. The crosshair symbol could be used to assist hover by aligning the centre of the cross hair with the ' $\mathrm{W}$ ' of the gull wing on the attitude indicator (Fig. 3).

3. The landing zone was designed with unprepared landing sites in mind. The landing zone provided the pilot with more ground perspective and the circular design allowed for different angles of approach to be made when accounting for wind direction. The central point provided an easily locatable target for which to align the flight path vector.

4. 3D augmented reality 'pyramids' around the front of the landing pad provided visual references for the pilot, 
Fig. 1 Semi-circle landing symbology in the HUD, clear sky

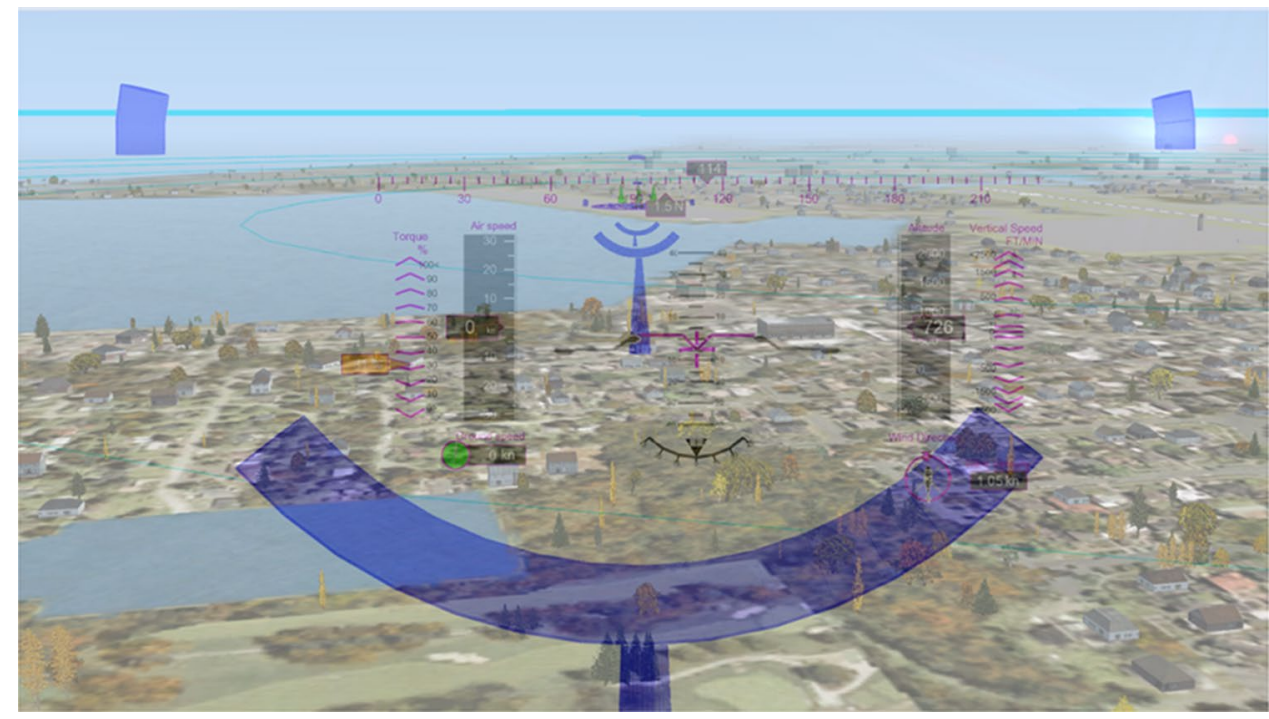

Fig. 2 Ground speed and drift indicator
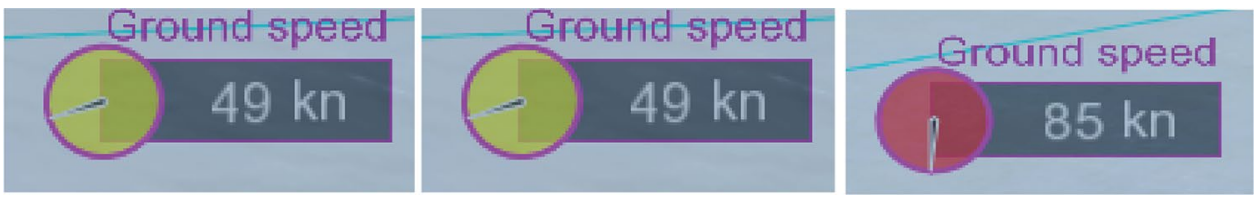

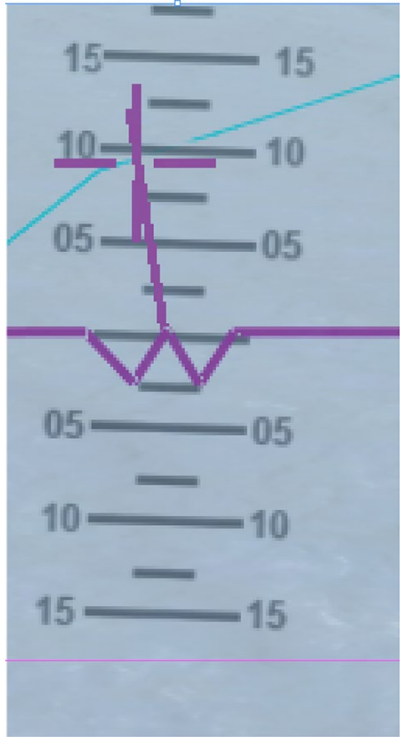

Fig. 3 Drift indicator showing forward movement

providing a sense of speed, direction and altitude. The smaller pyramids were $75 \mathrm{ft}$ high and the larger pyramids $150 \mathrm{ft}$ high, providing the pilot with additional visual cues. When the shorter pyramids directly align with the larger ones the pilot can be sure he is directly over the centre of the landing site (number 4, Fig. 4).

5. The flight path vector acted as a touch down indicator, became visible to the pilot when losing altitude, and represented the point on the ground the aircraft would hit if the current velocity was maintained (number 5 , Fig. 4).

\subsubsection{Questionnaires}

Two questionnaires were administered to participants in order to collect subjective ratings of situation awareness and workload, a Post-Landing Assessment (Stanton et al. 2016, 2017, 2019) and the Bedford Workload scale (Roscoe and Ellis 1990). The questionnaires were administered immediately following each simulated flight. As this study was interested in both the approach (transition down to hover) and landing (hover to aircraft on ground) phases, the questionnaires were administered twice, once for each phase. Pilots were instructed to define their own cut-off point between the approach and landing phases, however were requested to use the same cut-off point for all conditions. The chosen point was independent to each participant and not standardised to a set altitude or approach.

The Post-Landing Assessment Questionnaire was developed by SMEs at a leading rotary-wing aircraft manufacturer, and has been used within previous research (Stanton et al. 2016, 2017, 2019). The questionnaire required participants to rate their awareness of various flight parameters (e.g. desired heading, desired rate of descent) from 1 (low) to 7 (high). This questionnaire therefore offered a measure of perceived subjective situation awareness. In addition to assessing situation awareness, the Post-Landing Assessment 
Fig. 4 Landing symbology in the HUD, clear sky condition (the number relates to description in the main text)

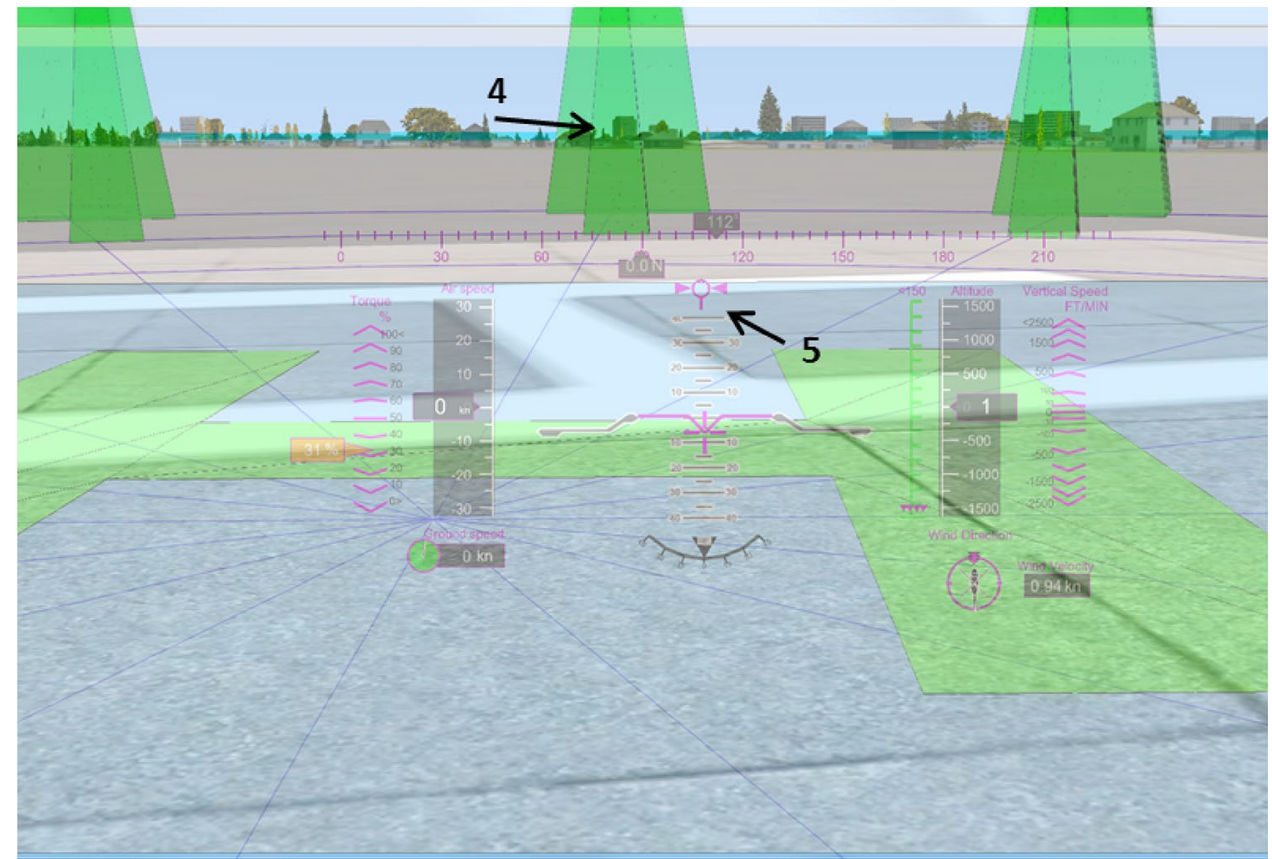

Questionnaire also asked for a rating of landing confidence, as measured by inclination to perform a go-around manoeuvre from 1 (not likely) to 7 (very likely).

The Bedford Workload Rating Scale (Roscoe and Ellis 1990) is a unidimensional mental workload assessment technique developed to assess pilot workload. Participants followed a decision tree to derive a workload rating for the task under analysis. A scale of 1 (low workload-workload insignificant) to 10 (high workload-task abandoned) was used.

\subsection{Procedure}

Prior to the start of the study, participants were briefed and presented with a consent form and demographic questionnaire. Participants completed an initial familiarisation session with the simulator to ensure that data gathered as part of the empirical trials were not influenced by confusion related to basic controls. Participants were also familiarised with the HUD in a talk through provided by the software developer who explained each instrument and the displayed symbology. Participants practised flying with the HUD prior to the start of the study. The familiarisation session lasted approximately $25 \mathrm{~min}$, with participants encouraged to ask any questions they had regarding the HUD or the simulator during this time. Following familiarisation, participants independently flew each of the four experimental conditions (clear, clear + HUD, degraded and degraded + HUD). The presentation order of the different conditions was counterbalanced. For each trial, participants started at $2 \mathrm{~nm}$ out to sea, at an altitude of 1000 feet and at a speed of 85 knots, which set the pilot up on a $4^{\circ}$ glideslope. Participants were instructed to fly to the runway and land the aircraft. Following completion of each trial, participants completed the required questionnaires for both descent and landing. Participants were instructed to base their ratings purely on the scenario under evaluation and the HUD symbology. Once a participant had completed all four trials, the research team debriefed him.

\subsection{Analysis}

When choosing factors to include in each statistical model, family wise error rates were considered and collinearity examined. Bedford workload measurements and confidence scores were examined in separate models. Firstly, $2 \times 2 \times 2$ within-subjects multivariate analyses of variance (MANOVAs) examined whether weather conditions (clear/fog), display type (no HUD/HUD) and flight phase (descent/landing) impact upon pilot's subjective situational awareness ratings. Univariate analyses of variance (ANOVAs) were conducted for further evaluation of dependant variables, including the subscales of the PostLanding Assessment Questionnaire alongside post hoc tests where appropriate. To account for multiple comparisons the Bonferroni correction method was used for all analysis. It should be noted that further analysis was continued despite the main MANOVA model failing to reach overall significance. This decision was made due to the limited sample size for this study, primarily a consequence of the nature of participants, as experienced rotary-wing pilots, high effect sizes identified and exploratory nature 
of this work. Caution is therefore warranted when considering the larger analysis. Consequently, findings will be presented graphically and results will be discussed primarily in terms of mean differences between weather (Clear or Fog) and symbology (HUD or No HUD). For readers interested in the full analysis, the results of statistical tests are presented separately within the supplementary material for the current paper.

\section{Results}

For complete data analysis please consult the supplementary resources available with this paper. As a summary of the overall data, means and standard deviations for all variables considered within the study are presented within Table 1 and main effect outputs from the statistical tests are presented in Table 2.

Table 1 Means and stand deviations of pilots' Post-Landing Assessment, Bedford Workload Scores during descent and landing phases of flight

\begin{tabular}{|c|c|c|c|c|c|c|c|c|}
\hline & \multicolumn{4}{|l|}{ Mean \pm SD } & \multicolumn{4}{|l|}{ Mean \pm SD } \\
\hline & \multicolumn{4}{|c|}{ Descent phase } & \multicolumn{4}{|c|}{ Landing phase } \\
\hline & \multicolumn{2}{|l|}{ Clear } & \multicolumn{2}{|l|}{ Fog } & \multicolumn{2}{|l|}{ Clear } & \multicolumn{2}{|l|}{ Fog } \\
\hline & No HUD & HUD & No HUD & HUD & No HUD & HUD & No HUD & HUD \\
\hline \multicolumn{9}{|l|}{ Awareness } \\
\hline Heading & $5.00 \pm 1.97$ & $5.39 \pm 1.58$ & $4.44 \pm 1.76$ & $5.50 \pm 1.15$ & $4.94 \pm 1.80$ & $5.06 \pm 1.70$ & $2.17 \pm 0.99$ & $5.06 \pm 1.73$ \\
\hline Rate of descent & $4.67 \pm 1.53$ & $5.11 \pm 1.57$ & $3.78 \pm 1.56$ & $4.72 \pm 1.56$ & $4.44 \pm 1.58$ & $4.44 \pm 1.79$ & $3.00 \pm 1.71$ & $4.22 \pm 1.63$ \\
\hline Ground speed & $3.72 \pm 1.56$ & $4.28 \pm 1.84$ & $3.44 \pm 1.46$ & $3.67 \pm 1.53$ & $4.33 \pm 1.57$ & $4.44 \pm 1.65$ & $2.61 \pm 1.42$ & $4.83 \pm 1.54$ \\
\hline Power status & $4.28 \pm 1.81$ & $4.28 \pm 1.74$ & $3.56 \pm 1.46$ & $3.94 \pm 1.59$ & $3.89 \pm 1.88$ & $4.11 \pm 1.64$ & $3.00 \pm 1.64$ & $3.72 \pm 1.81$ \\
\hline Landing & $5.50 \pm 1.25$ & $5.78 \pm 1.00$ & $2.39 \pm 1.46$ & $5.89 \pm 0.96$ & $5.17 \pm 1.50$ & $5.56 \pm 1.62$ & $1.78 \pm 1.26$ & $5.78 \pm 1.40$ \\
\hline Drift & $4.11 \pm 1.45$ & $4.22 \pm 1.96$ & $2.56 \pm 1.65$ & $4.22 \pm 1.66$ & $4.39 \pm 1.50$ & $4.56 \pm 1.76$ & $2.22 \pm 1.66$ & $4.28 \pm 1.36$ \\
\hline Outside environment & $5.56 \pm 1.46$ & $4.67 \pm 1.61$ & $2.83 \pm 1.54$ & $3.61 \pm 1.79$ & $5.33 \pm 1.46$ & $4.17 \pm 1.86$ & $1.72 \pm 1.27$ & $3.39 \pm 1.75$ \\
\hline \multicolumn{9}{|l|}{ Bedford } \\
\hline WL & $4.17 \pm 1.95$ & $4.11 \pm 1.94$ & $6.33 \pm 1.41$ & $4.94 \pm 1.92$ & $4.89 \pm 1.64$ & $4.94 \pm 2.26$ & $8.00 \pm 1.33$ & $5.72 \pm 1.96$ \\
\hline Go around & $2.33 \pm 1.57$ & $2.78 \pm 2.18$ & $4.94 \pm 2.39$ & $2.61 \pm 2.25$ & $2.56 \pm 1.85$ & $2.00 \pm 1.24$ & $4.78 \pm 2.39$ & $3.50 \pm 2.60$ \\
\hline
\end{tabular}

Table 2 Statistical analysis of pilots Post-Landing Assessment and Bedford Workload Scale scores during descent and landing phases

\begin{tabular}{|c|c|c|c|c|c|c|c|c|c|c|c|c|c|c|}
\hline & \multicolumn{2}{|l|}{ Weather } & \multicolumn{2}{|l|}{ Display } & \multicolumn{2}{|l|}{ Phase } & \multicolumn{2}{|l|}{$\begin{array}{l}\text { Weather } \times \\
\text { display }\end{array}$} & \multicolumn{2}{|l|}{$\begin{array}{l}\text { Weather } \times \\
\text { phase }\end{array}$} & \multicolumn{2}{|c|}{$\begin{array}{l}\text { Display } \times \\
\text { phase }\end{array}$} & \multicolumn{2}{|c|}{$\begin{array}{l}\text { Weather } \times \text { dis- } \\
\text { play } \times \text { phase }\end{array}$} \\
\hline & $F$ value & $\eta p^{2}$ & $F$ value & $\eta p^{2}$ & $F$ value & $\eta p^{2}$ & $F$ value & $\eta p^{2}$ & $F$ value & $\eta p^{2}$ & $F$ value & $\eta p^{2}$ & $F$ value & $\eta p^{2}$ \\
\hline \multicolumn{15}{|l|}{ Awareness } \\
\hline Main effect & $7.37 * * *$ & 0.82 & $14.21 * * *$ & 0.90 & $7.06 * * *$ & 0.82 & $11.55^{* * *}$ & 0.88 & $3.38 *$ & 0.68 & 0.91 & - & 2.69 & - \\
\hline Heading & $6.63 *$ & 0.28 & $33.87 * * *$ & 0.67 & $17.65 * * *$ & 0.51 & $12.41 * * *$ & 0.42 & $34.71 * * *$ & 0.67 & 3.16 & - & $10.51 * * *$ & 0.38 \\
\hline Rate of descent & $18.56 * * *$ & 0.52 & $4.65^{*}$ & 0.21 & $10.68 * * *$ & 0.39 & $18.29 * * *$ & 0.52 & 0.30 & - & 0.08 & & 1.31 & - \\
\hline Ground speed & $8.73 * * *$ & 0.34 & $18.08 * * *$ & 0.52 & 1.28 & - & $4.37 *$ & 0.20 & 0.59 & - & $5.35 *$ & 0.24 & $10.99 * * *$ & 0.39 \\
\hline Power status & $10.04 * * *$ & 0.37 & $4.53 *$ & 0.21 & 1.86 & - & 1.03 & - & 0.18 & - & 1.34 & - & 0.04 & - \\
\hline Landing & $50.48 * * *$ & 0.75 & $91.72 * * *$ & 0.84 & 3.98 & - & $83.80 * * *$ & 0.83 & 0.06 & - & 0.86 & - & 0.43 & - \\
\hline Drift & $21.29 * * *$ & 0.56 & $12.43 * * *$ & 0.42 & 0.16 & - & $13.33 * * *$ & 0.44 & 2.33 & - & 1.11 & - & 0.18 & - \\
\hline Outside environment & $39.51 * * *$ & 0.70 & 0.07 & - & $15.22 * * *$ & 0.47 & $26.20 * * *$ & 0.61 & 0.85 & - & 0.91 & - & 3.24 & - \\
\hline \multicolumn{15}{|l|}{ Bedford workload } \\
\hline WL & $32.87 * * *$ & 0.66 & $8.68 * * *$ & 0.34 & $29.85 * * *$ & 0.64 & $13.81 * * *$ & 0.45 & 0.69 & - & 1.70 & - & 2.28 & - \\
\hline Go around & $22.79 * * *$ & 0.57 & $6.37 *$ & 0.27 & 0.02 & - & $5.16^{*}$ & 0.23 & 1.94 & - & 0.00 & - & 3.56 & - \\
\hline
\end{tabular}

$* p<0.05, * * p<0.01, * * * p<0.001$ 


\subsection{Post-Landing Assessment Questionnaire}

The Post-Landing Assessment comprised seven awareness ratings, heading, rate of descent, ground speed, power status, landing area, drift and outside environment. Trends from each of these metrics are discussed below.

\subsubsection{Heading}

Pilots' ratings of awareness of heading for descent (left) and landing (right) phases of flight are presented within Fig. 5. As can be seen for both phases of descent and landing, fog acted to reduce pilots' awareness of heading when no HUD was available, this finding was most apparent when considering the landing stage, which saw a dramatic reduction in awareness of heading ratings. Awareness of heading did not differ however when pilots had access to the HUD, in fact a marginal improvement can be seen when pilots were descending within the fog condition and had access to the HUD, suggesting that use of the HUD facilitated rather than hindered awareness.

\subsubsection{Rate of descent}

Pilots' ratings of awareness of rate of descent for both descent (left) and landing (right) phases of flight are presented within Fig. 6. Of immediate notice is that the HUD facilitated pilots' awareness of rate of descent within clear condition for both descent and landing phase of flight. Whist the descent phase showed a slight degradation of awareness of rate of descent during fog for both HUD and no HUD conditions, this was more apparent within the No HUD condition. When considering the landing phase, degradation of awareness was only seen within the No HUD condition. It is clear from this figure that the HUD consistently benefited pilots' awareness of rate of descent.
Fig. 5 Pilots' awareness of heading for descent (left) and landing (right) phases of flight. Awareness of heading was greatly impeded during the landing phase in fog when participants did not have access to the HUD



Fig. 6 Pilots' awareness of rate of descent for both descent (left) and landing (right) phases of flight
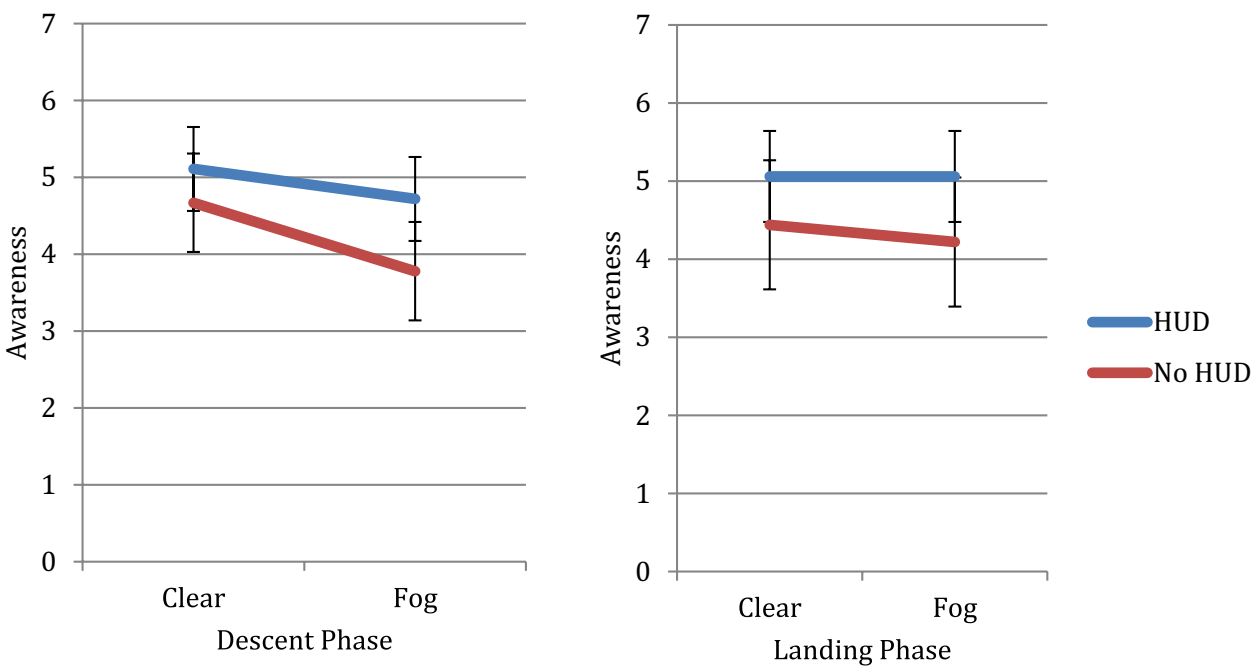


\subsubsection{Ground speed}

Figure 7 presents pilots' ratings of awareness of ground speed for both descent (left) and landing (right) phases. Whilst it appeared that the HUD offered limited benefited within the descent phase, clear advantage could be seen within the landing phase. During the landing phase, when pilots' had access to the HUD, their awareness of ground speed was higher within fog than during clear conditions. In contrast, without the HUD, pilots' awareness of ground speed was dramatically reduced. This suggests that the HUD was beneficial to pilots' awareness of ground speed only during the final landing phase, but manifested in a noticeable improvement in this phase.

\subsubsection{Power status}

Figure 8 presents pilots' ratings of awareness of power status for both descent (left) and landing (right) phases of flight. Whilst awareness of power status was impeded by fog during both phases of flight, impediment was a greater when participants did not have access to the HUD. Weather was of greater effect during the landing stage of flight, whereby a greater negative effect of degraded visibility was seen. It should be noted that even when pilots had access to the HUD awareness of power status was still impeded suggesting further refinement and development of the HUD are needed to minimise the disruption that fog caused this metric.

\subsubsection{Landing area}

Pilots' awareness of landing area for both the descent phase and landing phase is presented in Fig. 9. As can be seen,
Fig. 7 Pilots' awareness of ground speed for both descent (left) and landing (right) phases of flight. The HUD was of greater benefit during the landing phase
Fig. 8 Pilots' awareness of power status during descent (left) and landing (right) phases of flight

Fig. 9 Pilots' awareness of landing area during both descent (left) and landing (right) phases of flight. As can be seen access to the HUD was of considerable benefit to pilots when faced with fog during both phases



access to the HUD facilitated pilots' awareness of landing area, and awareness of landing area actually increased within the fog condition when the HUD was being used relative to clear conditions. In contrast, when pilots' did not have access to the HUD awareness of landing area was dramatically reduced within the fog condition. These trends were consistent for both descent and landing phases suggesting that the HUD was of consistent benefit to the pilots during the final phases of flight.

\subsubsection{Drift}

Pilots' awareness of drift for descent (left) and landing (right) phases is presented in Fig. 10. Although awareness of drift does appear to be somewhat lower than other metric, it can be seen fog acted to dramatically reduce pilots' awareness of drift during both stages of flight. Access to the HUD allowed pilots' to maintain awareness of drift to a comparable level seen during clear and non-degraded flight, suggesting that the HUD supported Pilot's needs.

\subsubsection{Outside environment}

Pilot's awareness of the outside environment for descent (left) and landing (right) phases is presented within Fig. 11. Of key interest within this figure is that access to the HUD impeded rather than facilitated pilots' awareness during both descent and landing phases when conditions were clear. This can be compared to both phases within degraded weather conditions whereby it was seen that the HUD was of considerable benefit to pilots' awareness of the outside environment during both phases of flight.
Fig. 10 Pilots' awareness of drift during both descent (left) and landing (right) phases of flight. Access to the HUD was beneficial to pilots when faced with fog during both phases
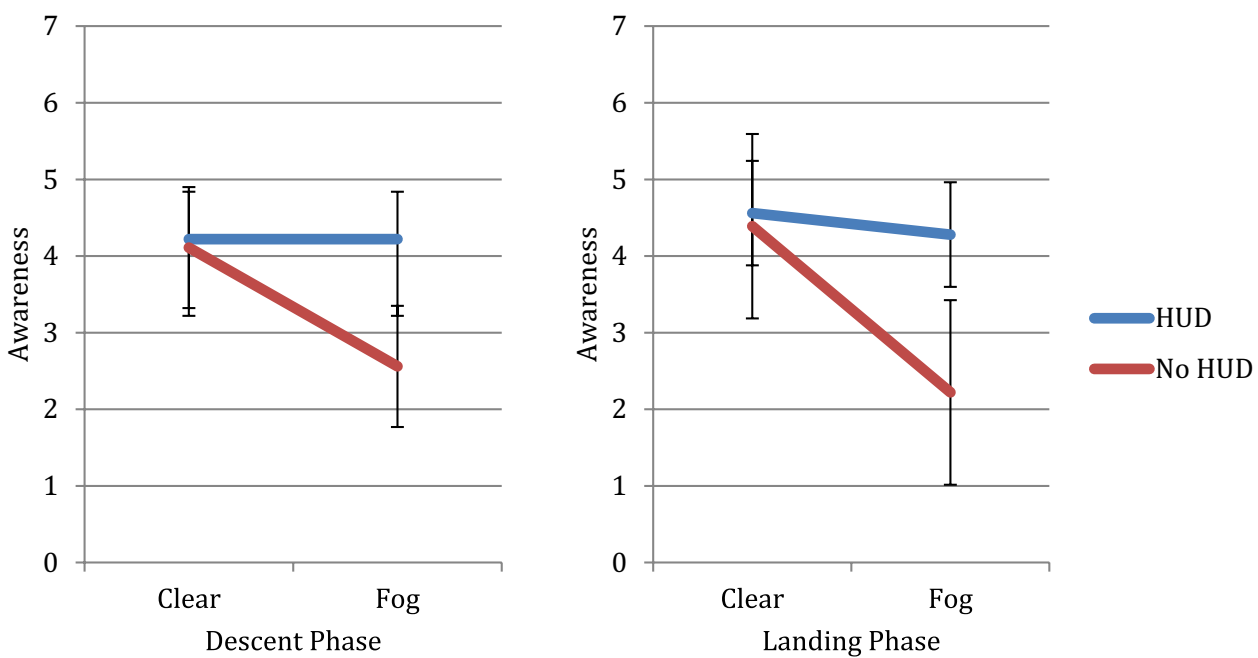
Fig. 11 Pilots' awareness of outside environment during both descent (left) and landing to the HUD was of benefit to pilots when faced with fog during both phases, however acted to impede awareness within clear conditions (right) phases of flight. Access


Fig. 12 Pilots' likelihood to complete a go-around manoeuvre during both descent (left) and landing (right) phases of flight. Access to the HUD reduced participants' likelihood to complete a go-around maneuverer during both phases
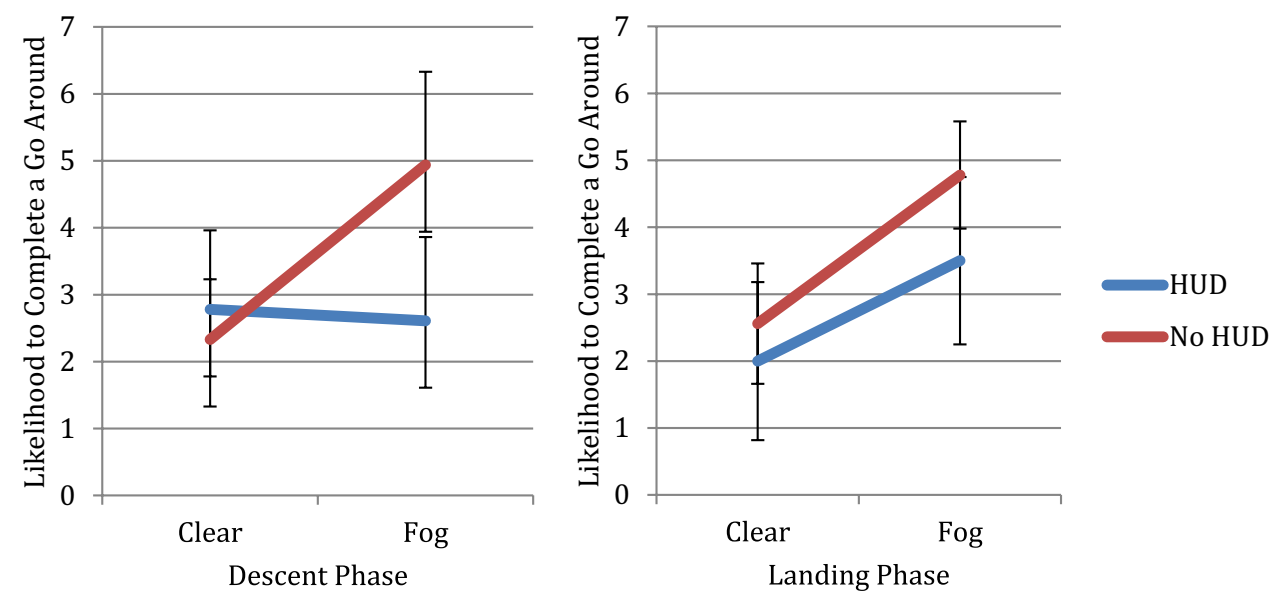

\subsection{Likelihood to complete a go around}

Pilot's likelihood to complete a go-around manoeuvre for descent (left) and landing (right) phases is presented within Fig. 12. It appears that access to the HUD was of greatest benefit during the descent stage of flight, allowing pilots to maintain the same confidence as within clear environments. Within the landing phase, it was seen that although access to the HUD was of benefit, degraded visibility still increased pilots' likelihood to complete a go around.

\subsection{Bedford workload}

Figure 13 presents pilots workload, for both descent and landing phases of flight as measured by the Bedford Workload Scale. As can be seen from this figure, operating within degraded visual environments (DVE) was associated with higher workload for both phases of flight. Access

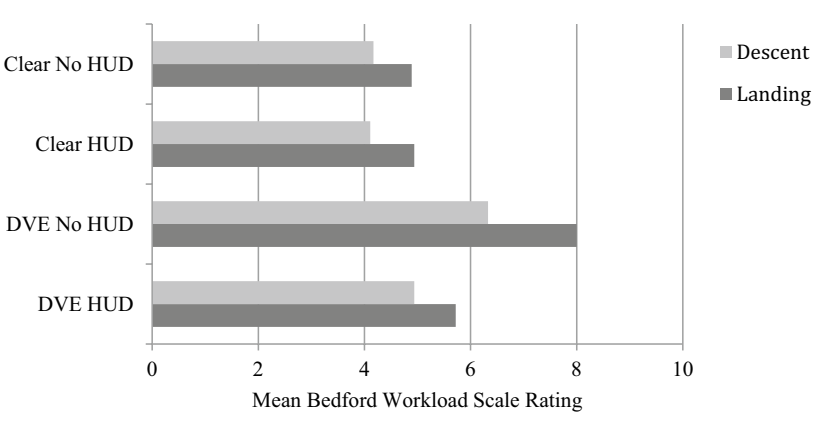

Fig. 13 Pilots workload, as measured by the Bedford Workload Scale for both descent and landing phases of flight

to the HUD however appears to dramatically reduce the workload associated with operating within such environments, resulting in workload rating highly comparable to operating within clear environments. 


\section{Discussion}

Results indicate that access to the HUD led to significant improvements to pilots' self assessed situation awareness and a reduction in pilot workload. The trend was for such improvements to be most prominent when comparing the use of the HUD in degraded, compared to clear visual conditions. The HUD did not reduce pilot situation awareness in degraded visual conditions when compared to no HUD in clear visual conditions, that is to say how pilots currently fly. Rather, awareness ratings were typically higher when such a comparison was made, particularly during the phase of landing. The workload ratings of pilots using the HUD in degraded visual conditions were similar to when using no HUD in clear visual conditions, indicating that the HUD supported pilots' needs whilst not unduly adding to workload.

Limited differences in pilot workload or situation awareness were observed with or without the HUD in clear visual conditions. Consistent with previous work examining fixed-wing aircraft (Lee and Liu 2003), pilot workload was significantly higher during the phase of landing, compared to the phase of descent. This can be clearly seen when considering that the lowest rating of situation awareness and highest workload ratings can be seen during the final phase of landing in degraded visual conditions without the HUD. Results shall now be discussed more specifically to evaluate the usefulness of the HUD.

\subsection{Weather}

Flight in degraded visual conditions significantly reduced pilot's awareness of all parameters and significantly increased pilot workload. The likelihood of a go around being performed was also significantly higher in degraded visual conditions. The results follow the same pattern as the previous work (Stanton et al. 2017, 2019), that is, conditions were degraded to an extent that a reliance on external information could not be maintained. In degraded visual conditions pilots must rely on cockpit instrumentation rather than external visual cues, the cognitive cost of which leads to increases in cognitive workload (Doehler et al. 2009; Prinzel et al. 2004).

\subsubsection{HUD in degraded visual conditions}

In degraded visual conditions access to the HUD significantly increased pilot's awareness of desired heading, rate of descent, groundspeed, power status and drift, the outside environment and landing site compared to when participants did not have access to the HUD. These results indicate that in degraded visual conditions the HUD was providing sufficient external visual cues to assist pilots when completing the flight task. Pilots' awareness of the various parameters was maintained to levels similar to those observed in clear visual conditions without the HUD, indicating that the presentation of a synthetic external environment allowed the pilots to continue to fly in an anticipatory fashion, and not shift to be reliant on cockpit instrumentation, typical with such conditions (Snow and French 2002; Harris 2011; Wickens 2002). As awareness of obstacles at the destination; sufficiency of visual cues for approach; stability of visual cues and sufficiency of visual aids for landing are primary performance factors for pilots during flight (Nascimento et al. 2013) it is clear that the HUD actively facilitated the pilots' task.

Pilots' workload significantly decreased when the HUD was used in degraded visual conditions, compared to flight within degraded visual environment with no HUD. The HUD was designed to preserve the most useful and unambiguous visual cues pilots naturally use for the flying task to ensure the intuitive processing of the presented information (Stanton and Plant 2010). The significant decrease in workload observed when pilots' had access to the HUD indicates that the information presented to pilots is both valuable and processed intuitively. The presentation of flight critical information an intuitive fashion acts to reduce cognitive workload (Foyle et al. 1992; Harris 2011; Prinzel et al. 2004; Ververs and Wickens 1998). The ability to access flight information without having to make large-scale redirections of visual gaze has been demonstrated to decrease workload (Snow and French 2002). The current results offer support to previous work which has demonstrated that the presentation of information in a HUD that does not require the diverting of cognitive resources into the cockpit, reduces workload and increases situation awareness (Snow and Reising 1999; Ververs and Wickens 1998).

\subsubsection{HUD in clear visual conditions}

The pattern of results in clear visual conditions with and without the HUD is similar to those observed in degraded visual conditions outlined. Participants' ratings of awareness generally increased when using the HUD in clear visual conditions, although this was not typically significant. Nevertheless, results are indicative that the HUD did not negatively influence the pilots' task and remained a useful aid, even in good visibility. It should be noted that pilot awareness of the outside environment did decrease when using the HUD in clear conditions, although this decrease was not statistically significant. Whilst previous research has demonstrated that HUDs can be detrimental to pilot situation awareness, particularly when task-irrelevant information is presented in demanding situations (Richards et al. 2016), the fact that 
pilots' awareness did not significantly decrease implies this was not problematic. The finding that HUDS have the potential to degrade awareness of the external environment can be linked to the issue of attentional tunnelling (Wickens and Alexander 2009; Fadden et al. 1998). This occurs when attention is allocated to a source of information for longer than is necessary, resulting in other pieces of information, which could be essential to task completion, being neglected or ignored (Wickens and Alexander 2009; Snow and French 2002). Given that in degraded conditions pilots' awareness of the outside environment was increased however, it is not necessarily that the HUD is inappropriately holding the attention of the pilots. This finding is however expressed with a measure of caution. Crawford and Neal (2006) in an exploration of HUDs and HDDs in fixed-wing aircraft found that whilst pilots reported highly positive reviews of the HUD, use of the HUD was associated with an increased number of missed events. Following an extensive literature review, Crawford and Neal (2006) suggest that many pilots are simply not aware of what they missed during flight operations, especially when using a HUD. Crawford and Neal draw upon the words of Hofer et al. (2001) to sum up their key finding "Pilots think they are seeing everything because all the information is being presented in their visual field when in fact they are not attending and processing everything" (p. 2) Previous research specific to the rotary-wing domain has demonstrated that access to conformal symbology, such as presented on the HUD within the current study, leads to faster detection response to environmental changes and improved flight path tracking accuracy (Fadden et al. 1998; Snow and French 2002). These contrasting findings highlight the need for future research. Future research is needed to examine the effectiveness of a de-clutter mode that removes elements of the HUD display during clear visual conditions. Such functionality has been demonstrated to be effective at facilitating both workload and performance of fixed-wing aircraft crews (Richards et al. 2016) however has not been applied to rotary-wing aircraft.

The current HUD was designed for use in both clear and degraded visual conditions. Given sufficient exposure and training, it is anticipated that pilots who routinely use the HUD, would not be influenced by changes to the external visual conditions during flight, as such, the impact of flight into degraded visual conditions would be reduced, minimising the impact of this event on no operational capability. Previous research has identified that low visibility during the critical phases of flight, primarily take-off and landing is a leading cause of delays at major commercial airports (Allan et al. 2001; Sridhar and Swei 2006). Furthermore, erroneous pilot decision making, including continued flight into degraded visual conditions contributed to $40 \%$ of rotary-wing accidents supporting gas operations in the Gulf of Mexico between 1983 and 2009 (Baker et al. 2011).
Access to a HUD, such as tested within the current study could reduce the impact of degraded visual conditions on delays and improve the safety of flight operations within such conditions.

\section{Limitations}

A notable limitation of the current research is a lack of analysis of the objective data. Whilst all participants were able to complete the descent and landing task investigated within the current study, observations of the research team indicated that the flight track paths flown by participants changed in the different weather conditions, with and without access the HUD. However, this data was not successfully captured and hence could not be analysed. Further research is therefore required to objectively examine how beneficial access to the HUD is in terms of changing recorded flight profiles and objective pilot performance. Potential applications of this work include the examination of flight economy, offering potential financial benefits to the helicopter operators as well as the potential to inform future training scenarios, facilitating safety. Such analysis is difficult due to the numerous suitable flight paths a rotary-wing pilot may fly, with flight paths varying both within and between participants. Seeking a way to understand and approach this research question remains topical when considering rotarywing operations. Further testing of the HUD is also required within a more advanced simulation facility. The current research was conducted in a simple desktop simulation facility, lacking in both the holistic appearance of a rotary-wing cockpit and also potentially low levels of immersion. Key to increased immersion is the impact of vibration conditions, when the pilots are provided with additional vestibular cues and additional challenges to overcome. Whilst it is anticipated that the findings of the current work would remain valid, understanding the nuance effects of vibrations would help to ensure that the identified findings remain credible within more realistic flight scenarios.

The lack of performance data for the current study is compounded by the number of variables considered, relative to the number of participants. As an exploratory piece of work, with a difficult to recruit population, it was deemed important to collect as much qualitative data as possible. However as a consequence of the limited sample this study had low statistical power. Although useful for understanding general trends in the data and providing initial feedback for the HUD concept and development, a significantly greater sample of participants would be needed to fully understand the complete impact of the HUD in different conditions.

Whilst we are exploring the potential for future technology, it should be reiterated that the technology considered within the current work is not mature enough to enter 
rotary-wing cockpits today. A clear issue that must be overcome is the impact of Motion Parallax, which could influence a pilot's depth perception, of clear central importance during the landing phase of operations. Although the technology is not of significant maturity today to fully augment the cockpit with eyes-out screens, initial work has started to explore the potential of colour HUDs, driven primarily within the automotive domain. Buckley and Stindt (2008) have presented initial work exploring colour HUDs, able to correct for curvature in the display surfaces. With increased desire for HUDs in divergent industries beyond aviation, it is not only possible, but also likely, that HUD displays will increase in capability, reduce in cost and be more widely accessible in the near future.

\section{Conclusions}

The operational benefits of rotary-wing aircraft are increasing demand for their use in a civilian setting, including for flight in degraded visual conditions. Previous research in the military and fixed-wing aviation domains have highlighted that, in degraded visual conditions, numerous task elements are impossible to perform without the aid of advanced equipment to facilitate such tasks (Swail and Jennings 1999). There is therefore a clear need for the development of future rotary-wing cockpit technologies to increase the operational capacity of civilian rotary-wing aircrafts in degraded visual conditions. The current study provides preliminary validation for the usefulness of a future cockpit technology in improving pilot situation awareness and reducing workload during flight in degraded visual conditions. Such improvements were analogous to flight in clear visual conditions using traditional HDD, indicating the HUD reduced the pilots need to switch their flight mode from being proactive to reactive. Moreover, the HUD also facilitated selfrated performance during clear visual conditions. The HUD design tested in the current study is at the early stages of product development, however, the current results offer encouragement for continued research concerning its usefulness and usability.

Acknowledgements The work was supported by funding from the EU 7th Framework project Grant no. ACP8-GA-2009-233682 ALICIA: All Condition Operations and Innovative Cockpit Architecture. We would like to thank the pilots who gave their time to participate in the study.

Open Access This article is distributed under the terms of the Creative Commons Attribution 4.0 International License (http://creativeco mmons.org/licenses/by/4.0/), which permits unrestricted use, distribution, and reproduction in any medium, provided you give appropriate credit to the original author(s) and the source, provide a link to the Creative Commons license, and indicate if changes were made.

\section{References}

Alexander AL, Wickens CD, Hardy TJ (2003) Examining the effects of guidance symbology, display size, and field of view on flight performance and situation awareness. In: Proceedings of the Human Factors and Ergonomics Society annual meeting, vol 47(1), pp 154-158. Sage Publications

Allan SS, Beesley JA, Evans JE, Gaddy SG (2001) Analysis of delay causality at Newark International Airport. In: 4th USA/Europe air traffic management R\&D seminar. Santa Fe, New Mexico, USA, pp 1-11

Arthur JTJ, Bailey RE, Williams SP, Prinzel LJ, Shelton KJ, Jones DR, Houston VE (2017) Review of head-worn displays for the next generation air transportation system. Opt Eng 56(5):051405

Bailey RE, Arthur JJ III, Williams SP (2004) Latency requirements for head-worn display S/EVS applications. In: Enhanced and synthetic vision 2004, vol 5424, pp 98-109. International Society for Optics and Photonics

Baker SP, Shanahan DF, Haaland W, Brady JE, Li G (2011) Helicopter crashes related to oil and gas operations in the Gulf of Mexico. Aviat Space Environ Med 82(9):885-889

Bell HH, Lyon DR (2000) Using observer ratings to assess situation awareness. In: Endsley MR (ed) Situation awareness analysis and measurement. Laurence Erlbaum Associates, Mahwah

Buckley E, Stindt D (2008) Full-colour holographic laser projector HUD. In: Proceedings of the SID annual symposium on vehicle displays, vol 15, pp 131-135

Crawford J, Neal A (2006) A review of the perceptual and cognitive issues associated with the use of head-up displays in commercial aviation. Int J Aviat Psychol 16(1):1-19

De Voogt AJ, Van Doorn RR (2007) Approaches and landings at wrong airports: analysis of 54 incidents and 11 accidents, 1981-2004. Aviat Space Environ Med 78(2):117-120

Doehler HU, Lüken T, Lantzsch R (2009) All Flight: a full scale enhanced and synthetic vision sensor suite for helicopter applications. In: SPIE defence, security, and sensing. International Society for Optics and Photonics, Florida, p 73280F

Endsley MR, Jones DG (2012) Designing for situation awareness: an approach to human-centered design, 2nd edn. Taylor and Francis, London

Fadden S, Ververs PM, Wickens CD (1998) Costs and benefits of headup display use: a meta-analytic approach. In: Proceedings of the Human Factors and Ergonomics Society 42nd annual meeting. Human Factors and Ergonomics Society, Santa Monica, pp 16-20

Federal Aviation Administration (2001) DOT Communication/Navigation/Surveillance, Engineering and Test Division, Weather Branch, ACT-320, Terminal Convective Weather Forecast (TCWF) 2000 Demonstration Report, 40 pp. and appendices

Foyle DC, Kaiser MK, Johnson WW (1992) Visual cues in lowlevel flight: implications for pilotage, training, simulation, and enhanced/synthetic vision systems. In: American Helicopter Society 48th annual forum, vol 1. Sage Publications, California, pp 253-260

Geise R, Enders A, Vahle H, Spieker H (2008) Scaled measurements of instrument-landing-system disturbances due to large taxiing aircraft. IEEE Trans Electromagn Compat 50(3):485-490

Harris D (2011) Human performance on the flight deck. Ashgate Publishing, Ltd., Aldershot

Hofer EF, Braune RJ, Boucek GP, Pfaff TA (2001) Attention-switching between near and far display domains: an exploratory study of pilots' attention switching ability with head-up and head-down tactical displays in simulated flight operations (Boeing IR\&D Document No. D6-36668). Boeing, Chicago 
Lee YH, Liu BS (2003) Inflight workload assessment: comparison of subjective and physiological measurements. Aviat Space Environ Med 74(10):1078-1084

McCarthy F, Budd L, Ison S (2015) Gender on the flightdeck: experiences of women commercial airline pilots in the UK. J Air Transp Manag 47:32-38

Melzer JE (2012) HMDs as enablers of situation awareness: the OODA loop and sense-making. In: Defense SPIE (ed) Security, and sensing. International Society for Optics and Photonics, Maryland, pp $83830 \mathrm{~F}-83830 \mathrm{~F}$

Nascimento FA, Majumdar A, Ochieng WY (2013) Investigating the truth of Heinrich's Pyramid in offshore helicopter transportation. Transp Res Rec J Transp Res Board 2336(1):105-116

Plant KL, Stanton NA (2012) Why did the pilots shut down the wrong engine? Explaining errors in context using schema theory and the perceptual cycle model. Saf Sci 50(2):300-315

Prinzel LLJ III, Kramer LJ, Comstock JR, Bailey RE, Hughes MF, Parrish RV (2002) NASA synthetic vision EGE flight test. In: Proceedings of the Human Factors and Ergonomics Society annual meeting, vol 46(1). Sage Publications, pp 135-139

Prinzel LJ III, Comstock JR Jr, Glaab LJ, Kramer LJ, Arthur JJ, Barry JS (2004) The efficacy of head-down and head-up synthetic vision display concepts for retro-and forward-fit of commercial aircraft. Int J Aviat Psychol 14(1):53-77

Rash CE (2000) Helmet-mounted displays: design issues for rotarywing aircraft. SPIE Press, Bellingham

Richards D, Scott S, Furness J, Lamb P, Jordan D, Moore D (2016) Functional symbology - evaluation of task-specific head-up display information for use on a commercial flight deck. In: AIAA modeling and simulation technologies conference Proceedings, AIAA modeling and simulation technologies conference, AIAA AVIATION forum. Held 13-17 June 2016. American Institute of Aeronautics and Astronautics, Washington, DC

Roscoe A, Ellis G (1990) A subjective rating scale for assessing pilot workload in flight. RAE, Farnborough

Shappell S, Wiegmann D (2004) HFACS analysis of military and civilian aviation accidents: a North American comparison. In: Proceedings of the annual meeting of the international society of air safety investigators, Gold Coast, Australia

Sneddon A, Mearns K, Flin R (2013) Stress, fatigue, situation awareness and safety in offshore drilling crews. Saf Sci 56:80-88

Snow MP, French GA (2002) Effects of primary flight symbology on workload and situational awareness in head-up synthetic vision display. In: Presented at the 21st digital avionics system conference (27-30 October, 2002). Wright Patterson Air Force Base, Ohio

Snow MP, Reising JM (1999) Effect of pathway-in-the-sky and synthetic terrain imagery on situation awareness in a simulated lowlevel ingress scenario. Air Force Research Laboratory Wright-Patterson AFB OH Crew System Interface Division. DTIC, Virginia

Sridhar B, Swei S (2006) Relationship between weather, traffic and delay based on empirical methods. In: 6th AIAA aviation technology, integration and operations conference (ATIO), Wichita, Kansas
Stanton NA, Plant KL (2010) Constraints analysis in the rotary-wing cockpit. A pilot study using cognitive work analysis. In: Proceedings of the international human computer interaction in aerospace conference 2010. Cape Canaveral, Florida

Stanton NA, Plant KL, Roberts AP, Harvey C, Thomas TG (2016) Extending helicopter operations to meet future integrated transportation needs. Appl Ergon 53:364-373

Stanton NA, Roberts AP, Plant KL, Allison CK, Harvey C (2017) Head-up displays assist helicopter pilots landing in degraded visual environments. Theor Issues Ergon Sci. https://doi. org/10.1080/1463922X.2017.1394506

Stanton NA, Plant KL, Roberts AP, Allison CK (2019) Use of highways in the sky and a virtual pad for landing head up display symbology to enable improved helicopter pilots situation awareness and workload in degraded visual conditions. Ergonomics 62(2):255-267

Swail C, Jennings S (1999) Enhanced and synthetic vision system concept for application to search and rescue missions. Fusion de données de senseur et l'intégration du facteur humain 12:3

Thomas LC, Wickens CD (2004) Eye-tracking and individual differences in off-normal event detection when flying with a synthetic vision system display. In: Proceedings of the Human Factors and Ergonomics Society annual meeting, vol 48(1). Sage Publications, New Orleans, pp 223-227

Ververs PM, Wickens CD (1998) Head-up displays: effect of clutter, display intensity, and display location on pilot performance. Int $\mathrm{J}$ Aviat Psychol 8(4):377-403

Ververs PM, Wickens CD (2000) Designing head-up displays (HUDs) to support flight path guidance while minimizing effects of cognitive tunneling. In: Proceedings of the Human Factors and Ergonomics Society annual meeting, vol 44(13). Sage Publications, Los Angeles, pp 45-48

Wickens CD (2002) Multiple resources and performance prediction. Theor Issues Ergon Sci 3(2):159-177

Wickens CD, Alexander AL (2009) Attentional tunneling and task management in synthetic vision displays. Int J Aviat Psychol 19(2):182-199

Williams D, Waller M, Koelling J, Burdette D, Doyle T, Capron W, (2001) Concept of operations for commercial and business aircraft synthetic vision systems. NASA technical memo. No. TM-2001211058. Langley, USA

Yeh M, Merlo JL, Wickens CD, Brandenburg DL (2003) Head up versus head down: the costs of imprecision, unreliability, and visual clutter on cue effectiveness for display signalling. Hum Factors J Hum Factors Ergon Soc 45(3):390-407

Publisher's Note Springer Nature remains neutral with regard to jurisdictional claims in published maps and institutional affiliations. 